\title{
Districting for routing with stochastic customers
}

\author{
Hongtao Lei • Gilbert Laporte • Bo Guo
}

Received: 26 September 2011/Accepted: 12 March 2012/Published online: 5 April 2012

(C) Springer-Verlag + EURO - The Association of European Operational Research Societies 2012

\begin{abstract}
We introduce the vehicle routing and districting problem with stochastic customers (VRDPSC). This problem is modelled and solved as a two-stage stochastic program during which the districting decisions are made in the first stage and the Beardwood-Halton-Hammersley formula is used to approximate the expected routing cost of each district in the second stage. District compactness is also considered as part of the objective function. We have developed a large neighbourhood search heuristic for VRDPSC. The heuristic was tested on modified Solomon instances and on modified Gehring and Homberger instances. Extensive computational results confirm the effectiveness of the proposed heuristic.
\end{abstract}

Keywords Districting - Stochastic vehicle routing - Large neighbourhood search metaheuristic

Mathematics Subject Classification 90B06 - 90B15

H. Lei $(\bowtie) \cdot$ B. Guo

College of Information Systems and Management, National University of Defense Technology, Changsha 410073, Hunan, People's Republic of China

e-mail: hongtaolei@yahoo.cn

B. Guo

e-mail: boguo@nudt.edu.cn

G. Laporte

Canada Research Chair in Distribution Management, HEC Montréal, 3000 chemin de la Côte-

Sainte-Catherine, Montreal H3T 2A7, Canada

e-mail: gilbert.laporte@cirrelt.ca

G. Laporte

Interuniversity Research Centre on Enterprise Networks, Logistics and Transportation (CIRRELT), Montreal, Canada 


\section{Introduction}

The problem considered in this paper is the vehicle routing and districting problem with stochastic customers (VRDPSC). It consists of designing the districts for a vehicle routing problem with stochastic customers with the aim of minimizing the expected cost of the solution. The problem is defined on an undirected graph $G=(V, E)$, where $V=\left\{v_{0}, \bar{V}, \widetilde{V}\right\}$ is the vertex set and $E=\left\{\left(v_{i}, v_{j}\right): v_{i}, v_{j} \in\right.$ $V, i<j\}$ is the edge set. Vertex $v_{0}$ is a depot at which are based several identical vehicles, $\bar{V}$ is the set of deterministic (regular) customers and $\widetilde{V}$ is a set of the stochastic customers whose locations and presence in the solution are uncertain. Thus our setting differs from those of the probabilistic travelling salesman problem (Jaillet 1988; Laporte et al. 1994) and of the vehicle routing problem with stochastic customers (Gendreau et al. 1996) in which the potential locations of the stochastic customers are known a priori. A symmetric matrix of Euclidean travel times, equal to travel costs, is defined on $E$. The VRDPSC consists of designing several contiguous vehicle districts such that; (1) all customers (including regular and stochastic customers) within the same district are serviced by the same vehicle, (2) each customer vertex is visited once by one vehicle, (3) an average service time $s$ is incurred when visiting a vertex, (4) each vehicle route has a normal duration limit $h$, but overtime is paid at rate $\theta$ if its duration exceeds $h$, and (5) an objective function combining vehicle cost, routing cost and a district compactness measure is minimized. Because of the presence of stochastic customers, the duration of a route in a district is a random variable. It should be noted at this stage that our methodology applies to the extreme case in which $\widetilde{V}=\emptyset$, i.e., all customers are deterministic, but their number can be quite large. However, it was designed with stochastic applications in mind.

The VRDPSC arises in the operations of courier companies such as DHL, FedEx or UPS. Each driver is assigned a district containing a set of regular customers, but other occasional customers also arise on a stochastic basis. In such contexts it is desirable to consistently assign the same sets of customers to drivers, and hence to create stable districts, to improve service (Groër et al. 2009).

The VRDPSC can be modelled and solved as a stochastic mathematical program. The most common solution methodology for this class of problems is called a priori optimization, a concept initially proposed by Bertsimas et al. (1990) and applied by several authors to the field of vehicle routing (e.g. Bertsimas 1992; Gendreau et al. 1996; Laporte et al. 2002, 2010; Tan et al. 2007; Mendoza et al. 2010 and Lei et al. 2011). In a priori optimization, a first-stage solution consisting of a set of districts is first constructed the realizations of the random variables (presence or absence of stochastic customers) are then revealed. In the second-stage solution, a vehicle route is constructed in each district to serve all its regular and stochastic customers. Whenever the maximal duration of a route is exceeded, an overtime cost is incurred. We therefore solve a stochastic problem with recourse.

There exists a rich literature on districting. Most of it deals with deterministic problems. These include the drawing of political districts (Mehrotra et al. 1992; Bozkaya et al. 2003), the design of school districts (Ferland and Guénette 1990), the 
construction of police districts (D'Amico et al. 2002), districting for home-care services (Blais et al. 2003), the alignment of commercial territories (Skiera and Albers 1998; Drexl 1999); Kalcsics et al. 2005; Rí os-Mercado and Fernández 2009) and the solution of location-districting problems (Novaes et al. 2009).

Research on stochastic districting problems has mostly been conducted in the context of vehicle routing. Haugland et al. (2007) have considered the problem of designing districts for vehicle routing problems with stochastic demands. The demands are assumed to be uncertain at the time when the districts were made, and these are revealed only after the districting decisions are determined. A tabu search heuristic was provided for the problem. Carlsson and Delage (2011) have introduced a robust framework for distributing the load of a vehicle routing problem over a fleet of vehicles when the location of demand points and their distribution are not known with certainty. Carlsson (2011) has studied an uncapacitated stochastic vehicle routing problem in which vehicle depot locations are fixed and client locations in a service region are unknown, but are assumed to be independent and identically distributed samples from a given probability density function.

To our knowledge, this paper is the first to consider stochastic customers in the context of a joint vehicle routing and districting problem. Instead of explicitly determining the vehicle routes, we approximate their expected cost by means of the Beardwood-Halton-Hammersley theorem (Beardwood et al. 1959). We integrate this approximation within a large neighbourhood search heuristic for the districting phase.

The remainder of the paper is organized as follows: The mathematical formulation of the problem is described in Sect. 2. In Sect. 3, we provide the detailed description of the approximation of the expected cost of routing in a district. In Sect. 4, we define the compactness measure of a district. A large neighbourhood search metaheuristic for the problem is described in Sect. 5, followed by computational experiments in Sect. 6 and by conclusions in Sect. 7 .

\section{Mathematical modeling as a stochastic program}

The VRDPSC is modeled as a two-stage stochastic program. The first-stage solution is a decomposition of $\bar{V}$ into $m$ districts, $V_{1}, \ldots, V_{m}$, each corresponding to a single vehicle route. A feasible district plan $x=\left\{V_{1}, \ldots, V_{m}\right\}$ must satisfy three conditions: (1) $v_{0} \in V_{k}(k=1, \ldots, m)$; (2) $\left\{V_{1} \backslash\left\{v_{0}\right\}, \ldots, V_{m} \backslash\left\{v_{0}\right\}\right\}$ is a partition of $\bar{V}$; (3) the district plan must induce a partition of the region into contiguous districts. After the first-stage solution has been computed, the sets $\widetilde{V}_{k}$ of stochastic customers are revealed and, in the second-stage solution, the cost of a vehicle route on $\left\{v_{0}\right\} \cup \bar{V}_{k} \cup \widetilde{V}_{k}$ is computed for each district $k$, where $\bar{V}_{k}=V_{k} \backslash\left\{v_{0}\right\}$. The workload $W_{k}$ of district $k$ is approximated as the expected length of an optimal traveling salesman problem (TSP) tour over $\bar{V}_{k} \cup \widetilde{V}_{k}$, plus twice the travel time between $v_{0}$ and the vertex of $\bar{V}_{k}$ closest to $v_{0}$. The number $m$ of districts is a decision variable.

The VRDPSC consists of computing 


$$
\min _{x} F(x)=\alpha_{\mathrm{m}} m+\alpha_{\mathrm{erc}} F_{\mathrm{erc}}+\alpha_{\mathrm{comp}} F_{\mathrm{comp}}
$$

such that $x=\left\{V_{1}, \ldots, V_{m}\right\}$ is a feasible districting plan. The objective function is a linear combination of three terms weighted by non-negative user-defined parameters $\alpha_{m}, \alpha_{\text {erc }}$ and $\alpha_{\text {comp }}$. The first term is the number of vehicles. The second term is the total expected routing cost. The third term is a compactness measure of the districts. The computations of $F_{\text {erc }}$ and $F_{\text {comp }}$ are detailed in Sects. 3 and 4, respectively. Contiguity is enforced through the construction and search mechanisms described in Sect. 5 .

\section{Approximation of the expected routing cost in a district}

Computing the expected routing cost of a given district requires the solution of a travelling salesman problem (TSP) over all deterministic and stochastic customers. We use the Beardwood-Halton-Hammersley theorem (Beardwood et al. 1959) to approximate this cost. It provides an asymptotic result based on identical distributions of customers over a compact area, which is not always the case in practice, and therefore its application may result in inaccuracies. However, it is sometimes used in strategic models (e.g. Carlsson 2011) which do not require detailed inputs.

Theorem 1 Let $\left\{X_{1}, \ldots, X_{n}\right\}, n \geq 1$, be a set of random variables in $\mathbb{R}^{\mathrm{dim}}$, independently and identically distributed with compact support. Then the length $L^{*}$ of a shortest travelling salesman tour through the points $X_{i}$ satisfies

$$
L^{*} / n^{(\operatorname{dim}-1) / \operatorname{dim}} \rightarrow \beta_{\operatorname{dim}} \int_{\mathbb{R}^{\operatorname{dim}}} f(x)^{(\operatorname{dim}-1) / \operatorname{dim}} d x, \quad \text { with probability } 1 \text {, as } n \rightarrow \infty,
$$

where $f(x)$ is the absolutely continuous part of the distribution of the $X_{i}$ and $\beta_{\operatorname{dim}}$ is a constant which depends on dim but not on the distribution.

Since our problem is defined in two dimensions, the optimal tour cost $L_{k}^{*}$ for district $k$ is

$$
L_{k}^{*} \approx \beta_{2} \sqrt{n_{k} A_{k}}
$$

where $A_{k}$ is the area of district $k, n_{k}=\bar{n}_{k}+\widetilde{n}_{k}$ is its number of customers, which includes the $\bar{n}_{k}$ regular customers and the $\widetilde{n}_{k}$ stochastic customers, and $\beta_{2}$ is a constant. The value of $\beta_{2}$ is truly asymptotic. Applegate et al. (2006), who have conducted extensive experiments, conclude that $\beta_{2}$ is empirically related to $n_{k}$, as shown in Table 1.

The workload of district $k$ can be calculated as

$$
W_{k}=2 d_{k}+\beta_{2} \sqrt{n_{k} A_{k}}+s n_{k},
$$

where $d_{k}$ is the shortest driving time between depot and the customer of district $k$ closest to the depot. The computation of (4) is distribution-dependent. For example, 
Table 1 Empirical value of $\beta_{2}$ as a function of $n_{k}$ (Applegate et al. 2006)

\begin{tabular}{ll}
\hline$n_{k}$ & $\beta_{2}$ \\
\hline 100 & 0.7764689 \\
200 & 0.7563542 \\
300 & 0.7477629 \\
400 & 0.7428444 \\
500 & 0.7394544 \\
600 & 0.7369409 \\
700 & 0.7349902 \\
800 & 0.7335751 \\
900 & 0.7321114 \\
1,000 & 0.7312235 \\
2,000 & 0.7256264 \\
\hline
\end{tabular}

assuming the number $\widetilde{n}_{k}$ of stochastic customers in district $k$ follows a Poisson $\left(\lambda_{k}\right)$ distribution, the expected cost of routing in district $k$ can be calculated as

$$
E\left(W_{k}^{+}\right)=\sum_{i=0}^{\infty} \frac{e^{-\lambda_{k}} \lambda_{k}^{i}}{i !}\left(2 d_{k}+\beta_{2} \sqrt{\left(\bar{n}_{k}+i\right) A_{k}}+s\left(\bar{n}_{k}+i\right)\right)^{+},
$$

where $(\cdot)^{+}=(\cdot)$ if $(\cdot) \leq h,(\cdot)^{+}=h+\theta((\cdot)-h)$ otherwise, and $\theta$ is the overtime rate. Therefore, the expected routing cost of solution $x$ is

$$
F_{\text {erc }}(x)=\sum_{k=1}^{m} E\left(W_{k}^{+}\right) \text {. }
$$

Note that the application of the Beardwood-Halton-Hammersley formula is particularly well suited to our problem since it uses no information on the precise location of the stochastic customers. We only require the distribution of their number to compute the expected routing cost.

\section{Compactness measure of a district}

As in Bozkaya et al. (2003), we use the following formula to measure the compactness of district:

$$
F_{\text {comp }}(x)=\frac{\sum_{k=1}^{m} B_{k}(x)-B}{2 B m}
$$

where $B_{k}(x)$ is the perimeter of district $k$ in solution $x$, and $B$ is the perimeter of the entire region. This formula computes the average normalized length of the inner boundaries of the districts. It is simple to implement and yields visually compact districts.

\section{Large neighbourhood search heuristic}

Since our problem embeds a stochastic TSP and should be solved for relatively large sizes, we have devised a large neighbourhood search heuristic for it. This 
type of heuristic was introduced by Shaw (1997) and has already been successfully applied to several routing problems (e.g. Shaw 1997, 1998; Ropke and Pisinger 2006; Pisinger and Ropke 2007; Goel and Gruhn 2008; Laporte et al. 2010; Lei et al. 2011; Hong 2012; and Ribeiro and Laporte 2012). This metaheuristic must be fine tuned to each application. In this section we describe its application to the VRDPSC.

Objective function

The objective function used in the heuristic search is the following:

$$
\min _{x} F(x)=\alpha_{m} m+\alpha_{\mathrm{erc}} F_{\text {erc }}+\alpha_{\text {comp }} F_{\text {comp }} .
$$

In our implementation, $\alpha_{m}$ is set to 1 and $\alpha_{\text {erc }}$ and $\alpha_{\text {comp }}$ are tested with different values.

Definition of the basic units

In order to operationalize the concept of contiguity, it is necessary to embed the regular customers in basic units which partition the region under study. Given a set of regular customer locations, we construct the basic units as follows: Assume that the location of customer $i$ in the instance region is described by the coordinates $\left(x_{i}, y_{i}\right)$. The region is defined by $\left[x_{\min }=\min _{i \in \bar{V}}\left\{x_{i}\right\}, x_{\max }=\max _{i \in \bar{V}}\left\{x_{i}\right\}\right]$ and $\left[y_{\min }=\min _{i \in \bar{V}}\left\{y_{i}\right\}, y_{\max }=\max _{i \in \bar{V}}\left\{y_{i}\right\}\right]$. It is partitioned into $n_{u}$ initial basic units, where $n_{u}=\left\lceil\left(x_{\max }-x_{\min }\right) / d\right\rceil \times\left\lceil\left(y_{\max }-y_{\min }\right) / d\right\rceil$ and $d=\min \left\{\min _{i, j \in \bar{V}}\left\{\mid x_{i}-\right.\right.$ $\left.\left.x_{j} \mid\right\}, \min _{i, j \in \bar{V}}\left\{\left|y_{i}-y_{j}\right|\right\}\right\}$. Any unit with no regular customer is merged with the nearest unit having at least one regular customer. Figure 1a shows the customers and depot in the plane on the instance mS-R-50 of Table 2. Figure 1b presents the generated initial basic units of instance mS-R-50. Figure 1c shows the final basic units of $\mathrm{mS}-\mathrm{R}-50$.

Given a set of basic units, which define a partition of the entire region, it is straightforward to use an adjacency list to indicate whether any two basic units are adjacent. Note that two neighbour units which only touch at a vertex are considered to be non-adjacent. To avoid creating the disconnected districts or enclaves, the construction heuristic as well as the removal and insertion operations comply with the following rules: (1) all exchange operations are performed on the boundary units of the districts; (2) operations that would disconnect some units from the remainder of the district are not performed.

\section{Construction of an initial solution}

We have devised the following construction heuristic to generate a good feasible initial solution. The heuristic first randomly selects a basic unit which includes at least one regular customer as the seed unit to initialize the first district. It gradually extends this district by adjoining to it the adjacent units yielding the least increase in the district workload. The expected workload of the extended district does not 


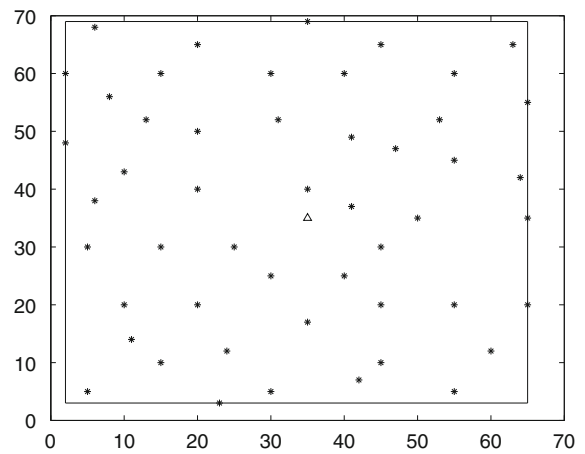

(a) Customers and depot in the plane

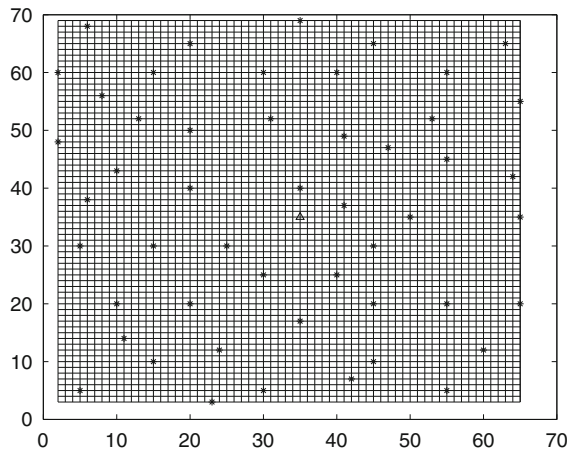

(b) Initial basic units

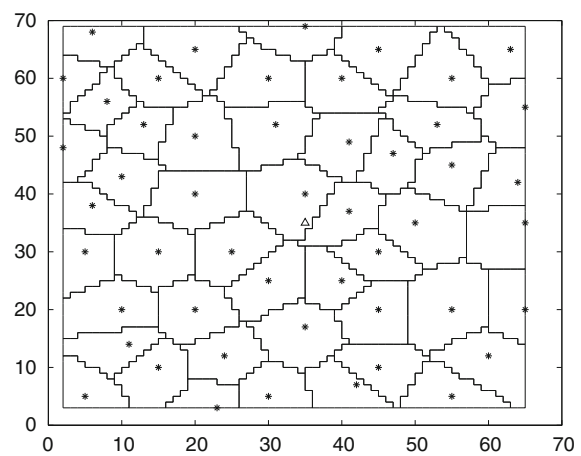

(c) Final basic units

Fig. 1 Generation of the basic units of instance mS-R-50 (a) Customers and depot in the plane. b Initial basic units. c Final basic units

Table 2 Modified Solomon instances

\begin{tabular}{llccc}
\hline Instance & Type & $|\bar{V}|$ & $E[|\widetilde{V}|]$ & $s$ \\
\hline $\mathrm{mS}-\mathrm{C} 1-50$ & $\mathrm{C} 1$ & 50 & 50 & 10 \\
$\mathrm{mS}-\mathrm{C} 2-50$ & $\mathrm{C} 2$ & 50 & 50 & 10 \\
$\mathrm{mS}-\mathrm{R}-50$ & $\mathrm{R}$ & 50 & 50 & 10 \\
$\mathrm{mS}-\mathrm{RC}-50$ & $\mathrm{RC}$ & 50 & 50 & 10 \\
$\mathrm{mS}-\mathrm{C} 1-75$ & $\mathrm{C} 1$ & 75 & 75 & 10 \\
$\mathrm{mS}-\mathrm{C} 2-75$ & $\mathrm{C} 2$ & 75 & 75 & 10 \\
$\mathrm{mS}-\mathrm{R}-75$ & $\mathrm{R}$ & 75 & 75 & 10 \\
$\mathrm{mS}-\mathrm{RC}-75$ & $\mathrm{RC}$ & 75 & 75 & 10 \\
$\mathrm{mS}-\mathrm{C} 1-100$ & $\mathrm{C} 1$ & 100 & 100 & 10 \\
$\mathrm{mS}-\mathrm{C} 2-100$ & $\mathrm{C} 2$ & 100 & 100 & 10 \\
$\mathrm{mS}-\mathrm{R}-100$ & $\mathrm{R}$ & 100 & 100 & 10 \\
$\mathrm{mS}-\mathrm{RC}-100$ & $\mathrm{RC}$ & 100 & 100 & 10 \\
\hline
\end{tabular}


exceed the duration limit $h$. If adjoining an adjacent unit would cause the expected workload to exceed $h$, this unit is not included in the district but serves as a seed unit for a new district. Any district with only one basic unit is eliminated and merged with the adjacent district so as to yield the lowest increased workload.

\section{Removal and insertion operators}

We now describe three removal operators and one insertion operator. At each iteration, $q$ boundary units are removed from their district using one of the three removal operators and are reinserted by means of an insertion operator, where $q$ is randomly selected in the interval $\left[\left\lceil 0.1 n_{\text {bou }}\right\rceil,\left\lceil 0.2 n_{\text {bou }}\right\rceil\right]$ as in Laporte et al. (2010), and $n_{\text {bou }}$ is the total number of the boundary basic units of current solution. The removal operators are randomly selected at each iteration and these remove-insert operators are combined to efficiently explore the solution space. Contiguity is always maintained. As in Bozkaya et al. (2003), we use a tabu tenure to avoid cycling: any removed basic unit is assigned a tabu status, i.e., it cannot be reinserted into its original district for $\phi$ iterations. As recommended by Haugland et al. (2007), $\phi$ is set equal to the number of districts in the initial solution. As usual, tabu move may be still performed if it yields a new incumbent solution.

\section{Long removal operator}

This operator concentrates on those districts with longer perimeters and are less likely to be compact. It works with a parameter $q$, as described at the beginning of Sect. 5.4

Step 0 . Set $\vartheta=0$ and $k=1$.

Step 1. Sort the districts in non-increasing order of the values of their perimeter. Step 2. If the number $m(x)$ of districts of the current solution $x$ is larger than $q$, randomly remove a boundary basic unit from each of the first $q$ districts without disconnecting it (i.e., while preserving contiguity) and stop.

Step 3. Randomly remove a boundary basic unit from district $k$ without disconnecting it.

Step 4. Set $\vartheta=\vartheta+1$ and $k=k+1$. If $k>m(x)$, set $k=1$. If $\vartheta=q$, stop; otherwise go to Step 3.

\section{Large removal operator}

This operator focuses on the districts with larger number of regular customers which are likely to have a larger workload.

Step 0 . Set $\vartheta=0$ and $k=1$.

Step 1. Sort the districts in non-increasing order of their number of regular customers. 
Step 2. If $m(x)>q$, remove the boundary basic unit with the largest number of regular customers from each of the first $q$ districts without disconnecting them and stop.

Step 3. Remove the boundary basic unit with the largest number of regular customers from district $k$ without disconnecting it.

Step 4. Set $\vartheta=\vartheta+1$ and $k=k+1$. If $k>m(x)$, set $k=1$. If $\vartheta=q$, stop; otherwise go to Step 3.

When each basic unit includes the same number of regular customers, the removed basic unit is randomly chosen from the boundary units with regular customers, without disconnecting the district.

\section{Random removal operator}

The operator randomly selects $q$ boundary basic units and removes them from their district without disconnecting it.

\section{Insertion operator}

This operator reinserts the removed units into the adjacent districts with the lowest increase in the objective function value.

Step 0 . Set $l=1$.

Step 1. Select a unit $u_{l}$ from the removed units and compute the increase in the objective function values when the selected unit is reinserted in an adjacent district.

Step 2. Choose a adjacent district yielding the lowest increase in the objective function value as the best district, while respecting the tabu tenure described in Sect. 5.5. If no feasible insertion district is found, go to Step 4.

Step 3. Insert the unit $u_{l}$ into the best district. If $l=q$, stop; otherwise set $l=l+1$ and go to Step 1 .

Step 4. Use $u_{l}$ to initialize a new district. Set $m(x)=m(x)+1$. If $l=q$, stop; otherwise set $l=l+1$ and go to Step 1 .

Acceptance and stopping criteria

We use the record-to-record travel (RRT) algorithm introduced by Dueck (1993) to define the acceptance criterion for a new solution. This works as follows: Assume $f^{*}$ is the value of the best current solution, called a record. The unique and positive parameter $\delta$ of the RRT algorithm is called a deviation. Let $x$ be a solution, $x^{\prime}$ a neighbour of $x$ and $f_{x^{\prime}}$ the objective value of solution $x^{\prime}$. Solution $x^{\prime}$ is accepted if $f_{x^{\prime}}<f^{*}+\delta$, and $f^{*}$ is updated if $f_{x^{\prime}}<f^{*}$. We set $\delta=0.1 f^{*}$. The search stops if solution quality has not improved for a given number of iterations or if a preset number of iterations have been executed. We set these values as 300 and 1,000, respectively, in our implementation. 
Summary of the large neighbourhood search heuristic

Our implementation of the large neighbourhood search heuristic can be summarized as follows:

Step 1. Initialize the parameters and use the construction heuristic to generate an initial solution. Set the objective value of the initial solution as the record and the best cost and compute the deviation. Set the initial solution as the best solution and define it as the current solution.

Step 2. Randomly select a removal operator from the three removal operators to remove $q$ boundary units from the current solution without disconnecting the districts. Then apply the insertion operator to repair the solution and to generate a new solution, respecting the tabu tenure mechanism.

Step 3. If the objective value of the new solution is smaller than the best cost, set the new solution as the best solution and set its objective value as the best cost. If the new solution is accepted using the RRT criterion, set it as the current solution. If the objective value of the new solution is smaller than the record, update the record and the deviation.

Step 4. Update the total number of the boundary units of current solution and update the value of $q$.

Step 5. If the stopping criterion is met, output the best solution and the best cost. Otherwise, go to Step 2.

\section{Computational experiments}

The algorithm described in Sect. 5 was coded using Matlab 7.0.4 and run on a laptop with $2 \mathrm{GHz}$ dual processor and $2 \mathrm{~GB}$ RAM. We now describe the results of extensive computational experiments.

We assume that all stochastic customers are Poisson distributed and the mean number $\lambda_{k}$ of stochastic customers of each district is equal to the number $\bar{n}_{k}$ of regular customers of the same district.

Experiments on the modified Solomon instances

We now describe a first set of experiments performed on the modified Solomon instances.

\section{Experimental design}

We have generated test instances derived from those of Solomon (1987). The coordinates of the regular customers are the same as in the Solomon instances, while the demands and time windows are not used. We consider six classes of instances: $\mathrm{R} 1, \mathrm{R} 2, \mathrm{C} 1, \mathrm{C} 2, \mathrm{RC} 1$ and $\mathrm{RC} 2$. The coordinates of R1 and R2 are the same, and so are those of RC1 and RC2. However, the coordinates of $\mathrm{C} 1$ and $\mathrm{C} 2$ are not identical. Hence we consider four types of instances: R, C1, C2 and RC. We choose the first 
50, 75 and 100 customer vertices as the regular customers in the tests. The service times of customers are equal to $10 \mathrm{~min}$, and $h$ is set to $480 \mathrm{~min}$.

Because there are no comparative data and no competing heuristic exist for our problem, comparisons with best known solutions are not possible. However, we can compare the initial solutions generated by our construction heuristic of Sect. 5.3 with the solutions obtained by our heuristic of Sect. 5. The detailed information of the modified Solomon instances tested is shown in Table 2.

\section{Computational results}

Table 3 presents computational results for the modified Solomon instances with $\alpha_{m}=1, \alpha_{\text {erc }}=1$ and $\alpha_{\text {comp }}=1$. The column "Construction heuristic" summarizes the results obtained from the construction heuristic of Sect. 5.3. The column "LNS heuristic" summarizes the results obtained by applying the heuristic of Sect. 5. The column " $m$ " gives the number of districts of the solutions. The column " $F_{\text {erc }}$ " presents the expected routing cost of the solutions, computed by Formula (6). The column " $F_{\text {comp" }}$ shows the compactness measure cost of the solutions, computed by Formula (7). The column " $F$ " is the total expected cost of the solutions, computed by Formula (8). We also report the total CPU time in seconds in the "Seconds" column for "LNS heuristic". The "Imp(\%)" column shows the percentage improvement in "Total cost" obtained by "LNS heuristic", compared with "Construction heuristic".

Table 3 Computational results on the modified Solomon instances

\begin{tabular}{|c|c|c|c|c|c|c|c|c|c|c|}
\hline \multirow[t]{2}{*}{ Instance } & \multicolumn{4}{|c|}{ Construction heuristic } & \multicolumn{5}{|c|}{ LNS heuristic } & \multirow[b]{2}{*}{$\operatorname{Imp}(\%)$} \\
\hline & $m$ & $F_{\text {erc }}$ & $F_{\text {comp }}$ & $F$ & $m$ & $F_{\text {erc }}$ & $F_{\text {comp }}$ & $F$ & Seconds & \\
\hline $\mathrm{mS}-\mathrm{C} 1-50$ & 4 & 1536.43 & 0.25 & 1540.68 & 4 & 1480.61 & 0.34 & 1484.95 & 87.08 & 3.62 \\
\hline $\mathrm{mS}-\mathrm{C} 2-50$ & 4 & 1685.23 & 0.25 & 1689.48 & 4 & 1579.45 & 0.35 & 1583.80 & 102.80 & 6.26 \\
\hline $\mathrm{mS}-\mathrm{R}-50$ & 6 & 1782.45 & 0.21 & 1788.66 & 4 & 1567.53 & 0.34 & 1571.87 & 136.86 & 12.12 \\
\hline $\mathrm{mS}-\mathrm{RC}-50$ & 6 & 2139.12 & 0.18 & 2145.30 & 4 & 1961.18 & 0.30 & 1965.48 & 161.33 & 8.38 \\
\hline 50-average & 5 & 1785.80 & 0.22 & 1791.02 & 4 & 1647.19 & 0.33 & 1651.52 & 122.02 & 7.59 \\
\hline $\mathrm{mS}-\mathrm{C} 1-75$ & 6 & 2674.04 & 0.21 & 2680.25 & 6 & 2437.72 & 0.26 & 2443.98 & 297.00 & 8.82 \\
\hline $\mathrm{mS}-\mathrm{C} 2-75$ & 6 & 2523.72 & 0.21 & 2529.93 & 6 & 2453.91 & 0.25 & 2460.16 & 169.31 & 2.76 \\
\hline mS-R-75 & 10 & 2537.26 & 0.17 & 2547.43 & 6 & 2273.95 & 0.27 & 2280.22 & 229.22 & 10.49 \\
\hline mS-RC-75 & 7 & 2745.37 & 0.18 & 2752.55 & 6 & 2560.07 & 0.30 & 2566.37 & 186.81 & 6.76 \\
\hline 75 -average & 7.25 & 2620.20 & 0.19 & 2627.54 & 6 & 2431.42 & 0.27 & 2437.69 & 220.59 & 7.21 \\
\hline $\mathrm{mS}-\mathrm{C} 1-100$ & 8 & 3366.82 & 0.19 & 3375.01 & 7 & 3216.03 & 0.23 & 3223.26 & 346.58 & 4.50 \\
\hline $\mathrm{mS}-\mathrm{C} 2-100$ & 10 & 3559.60 & 0.18 & 3569.78 & 7 & 3240.70 & 0.30 & 3248.00 & 347.95 & 9.01 \\
\hline $\mathrm{mS}-\mathrm{R}-100$ & 10 & 3217.65 & 0.18 & 3227.83 & 7 & 2895.51 & 0.26 & 2902.77 & 546.67 & 10.07 \\
\hline $\mathrm{mS}-\mathrm{RC}-100$ & 12 & 3624.82 & 0.15 & 3636.97 & 7 & 3228.23 & 0.26 & 3235.49 & 330.38 & 11.04 \\
\hline 100-average & 10 & 3442.22 & 0.17 & 3452.39 & 7 & 3155.69 & 0.26 & 3162.95 & 398.97 & 8.36 \\
\hline Average & 7.42 & 2616.04 & 0.19 & 2623.65 & 5.67 & 2411.43 & 0.29 & 2417.39 & 247.19 & 7.72 \\
\hline
\end{tabular}


Table 4 Modified Gehring and Homberger instances

\begin{tabular}{lllll}
\hline Instance & Type & $|\bar{V}|$ & $E[|\widetilde{V}|]$ & $s$ \\
\hline mGH-C1-150 & C1 & 150 & 150 & 10 \\
mGH-C2-150 & C2 & 150 & 150 & 10 \\
mGH-R1-150 & R1 & 150 & 150 & 10 \\
mGH-R2-150 & R2 & 150 & 150 & 10 \\
mGH-RC-150 & RC & 150 & 150 & 10 \\
mGH-C1-200 & C1 & 200 & 200 & 10 \\
mGH-C2-200 & C2 & 200 & 200 & 10 \\
mGH-R1-200 & R1 & 200 & 200 & 10 \\
mGH-R2-200 & R2 & 200 & 200 & 10 \\
mGH-RC-200 & RC & 200 & 200 & 10 \\
mGH-C1-300 & C1 & 300 & 300 & 10 \\
mGH-C2-300 & C2 & 300 & 300 & 10 \\
mGH-R1-300 & R1 & 300 & 300 & 10 \\
mGH-R2-300 & R2 & 300 & 300 & 10 \\
mGH-RC-300 & RC & 300 & 300 & 10 \\
mGH-C1-400 & C1 & 400 & 400 & 10 \\
mGH-C2-400 & C2 & 400 & 400 & 10 \\
mGH-R1-400 & R1 & 400 & 400 & 10 \\
mGH-R2-400 & R2 & 400 & 400 & 10 \\
mGH-RC-400 & RC & 400 & 400 & 10 \\
\hline
\end{tabular}

Table 3 clearly shows that the solutions of "LNS heuristic" are better than those of "Construction heuristic". The average improvement percentage in the total cost $F$ is $7.72 \%$. The average number of districts of the solutions of "LNS heuristic" is less than that of "Construction heuristic" and so is the average expected routing cost. However, the average compactness cost of the solutions of "LNS heuristic" is more than that of "Construction heuristic". The average CPU time for "LNS heuristic" is $247.19 \mathrm{~s}$.

Experiments on the modified Gehring and Homberger instances

We next present a second set of experiments performed on the modified Gehring and Homberger instances.

\section{Experimental design}

We have also generated test instances derived from those of Gehring and Homberger (1999). Like the modified Solomon instances, the coordinates of the Gehring and Homberger instances are used as the coordinates of the regular customers of the tested instances, and the demands, time windows and service times are not used. We consider six classes of instances: R1, R2, C1, C2, RC1 and RC2. And in the Gehring and Homberger instances, the coordinates of RC1 and RC2 are 


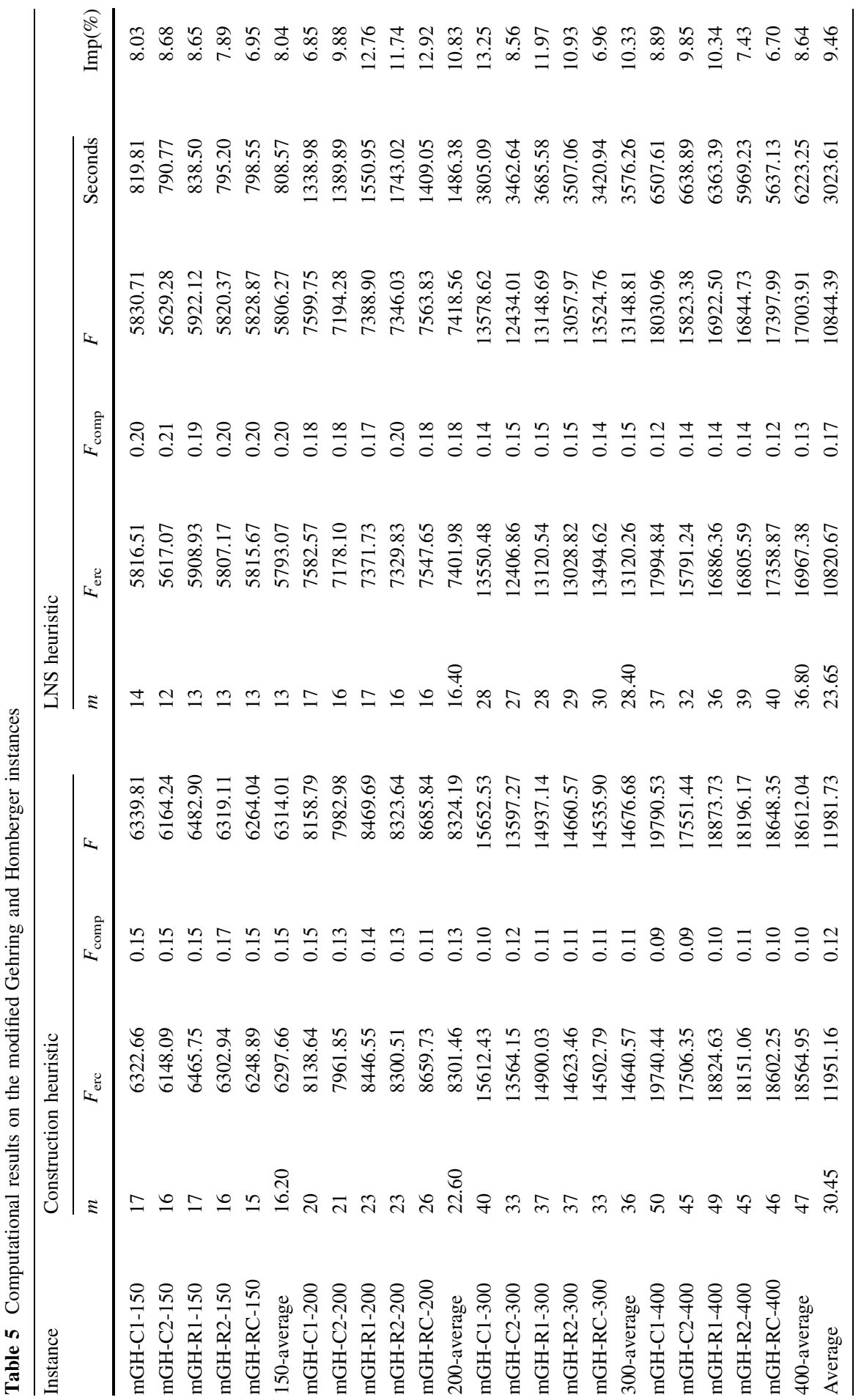




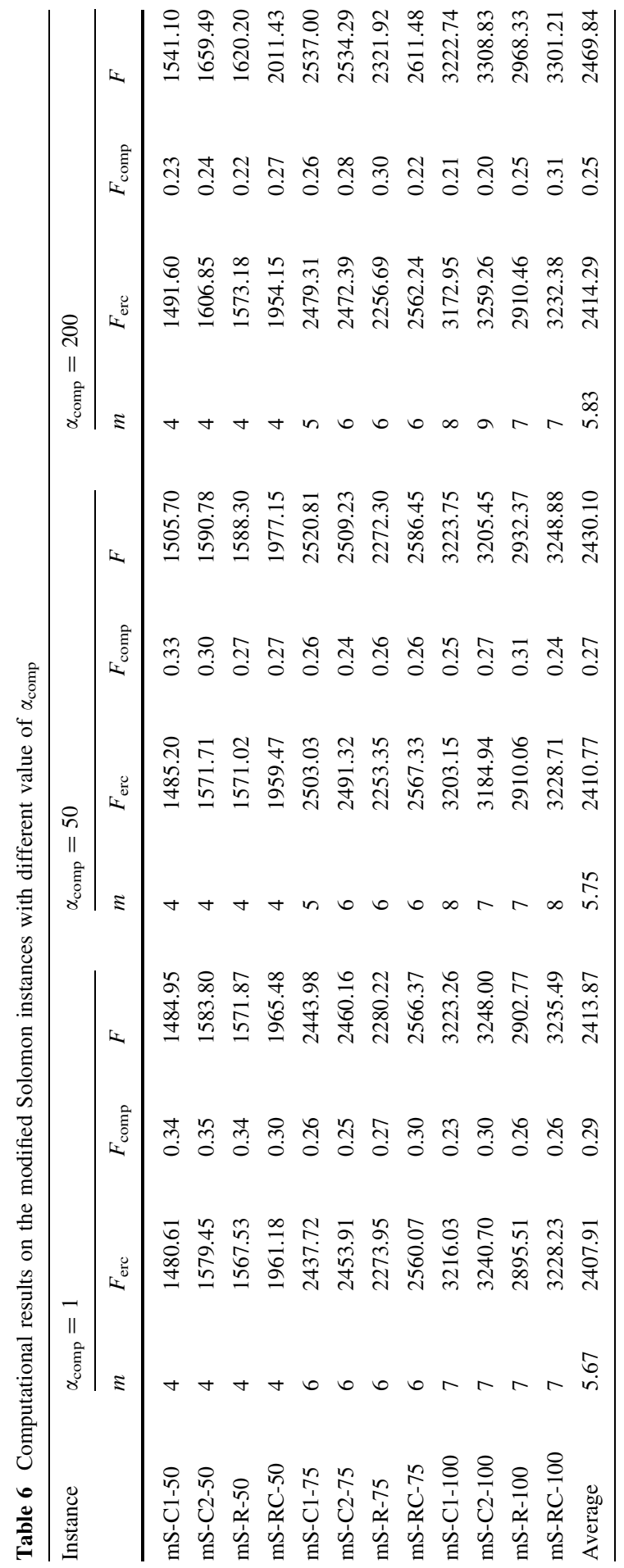


Fig. 2 Solution for instance mS-R-50 with $\alpha_{\text {comp }}=1$

Fig. 3 Solution for instance mS-R-50 with $\alpha_{\text {comp }}=50$

Fig. 4 Solution for instance mS-R-50 with $\alpha_{\text {comp }}=200$
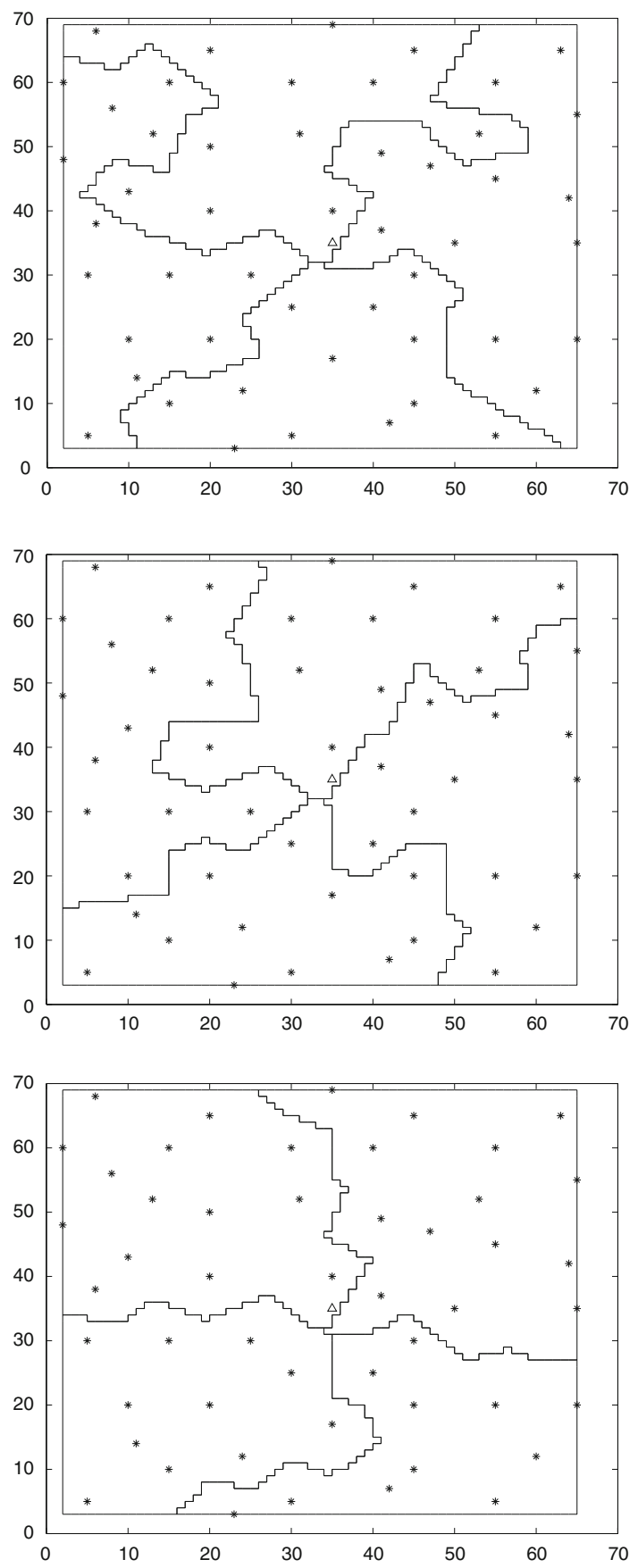

the same, but the coordinates of $\mathrm{C} 1$ and $\mathrm{C} 2$ are not identical and neither are those of R1 and R2. Hence we consider five types of instances: R1, R2, C1, C2 and RC. We, choose the 150, 200, 300 and 400 customer vertices as the regular customers in the 


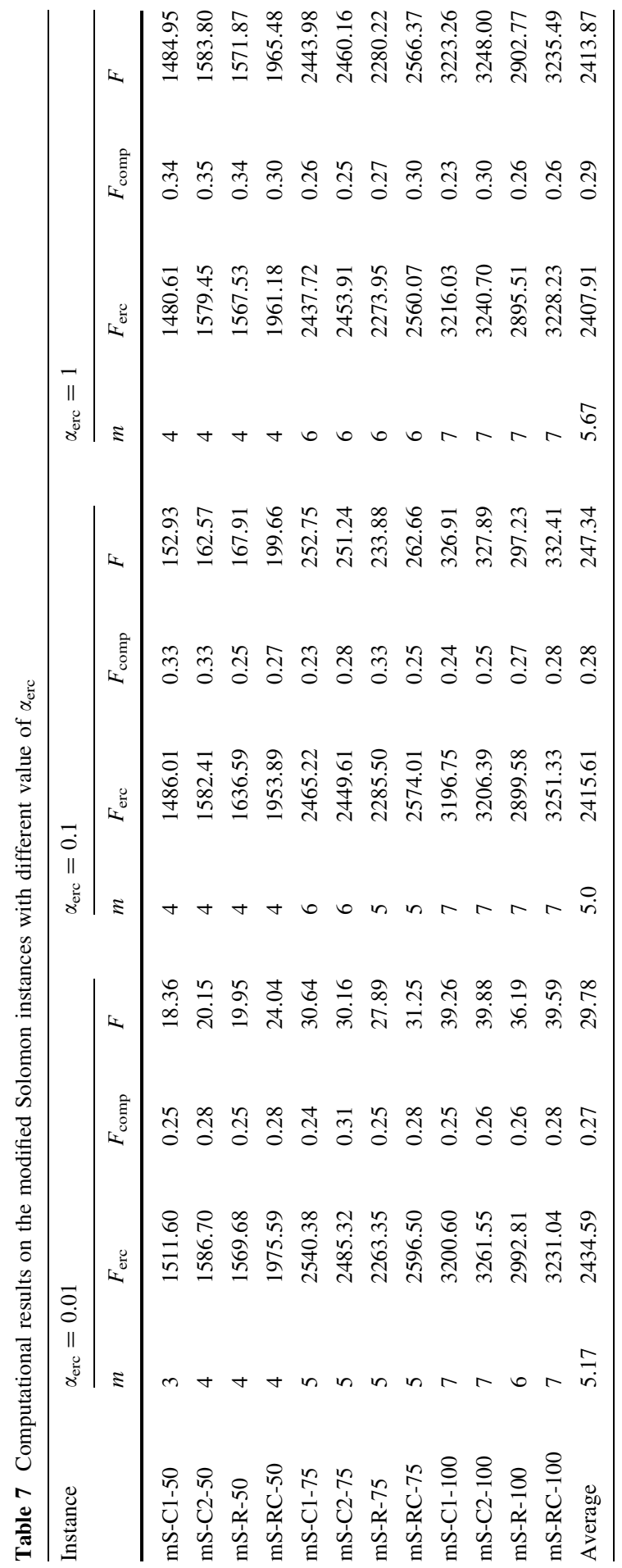


tests, and the regular customers of the instances with 150 regular customers are chosen from the first 150 customer vertices of the instances with 200 customers. The values of the service times of customers and the duration $h$ of each district are the same as those of the modified Solomon instances. The detailed information of the modified Gehring and Homberger instances tested is shown in Table 4.

\section{Computational results}

Table 5 shows the computational results for the modified Gehring and Homberger instances with $\alpha_{m}=1, \alpha_{\text {erc }}=1$ and $\alpha_{\text {comp }}=1$. It indicates that the solutions of "LNS heuristic" are better than those of "Construction heuristic". The average improvement percentage is $9.46 \%$. Similar to the results on the modified Solomon instances, the average number of districts of the solutions of "LNS heuristic" is less than that of "Construction heuristic", and so is the average expected routing cost, but the average compactness cost of the solutions of "LNS heuristic" is more than that of "Construction heuristic". The average CPU time for "LNS heuristic" is $3023.61 \mathrm{~s}$.

\section{Experiments with different parameters}

We have performed tests with different parameters by successively varying the multiplier $\alpha_{\text {comp }}$ of $F_{\text {comp }}$ and the multiplier $\alpha_{\text {erc }}$ of $F_{\text {erc }}$ in the objective function. Table 6 provides the solution values obtained with $\alpha_{\text {comp }}=1, \alpha_{\text {comp }}=50$ and $\alpha_{\text {comp }}=100$, leaving the other multipliers unchanged. As expected, when the value of $\alpha_{\text {comp }}$ becomes larger, the average value of $F_{\text {comp }}$ becomes smaller. In contrast, the average values of $m$ and $F_{\text {erc }}$ become larger. Figures 2, 3 and 4 show the districts of the final best solution of the instance mS-R-50 when $\alpha_{\text {comp }}=1, \alpha_{\text {comp }}=50$ and $\alpha_{\text {comp }}=100$, respectively. We can see that when the value of $\alpha_{\text {comp }}$ increases, the districts of the final best solution become more compact. Table 7 provides the comparison of the computational solutions with $\alpha_{\mathrm{erc}}=0.01, \alpha_{\mathrm{erc}}=0.1$ and $\alpha_{\text {erc }}=1$, leaving the other multipliers unchanged. Like in Table 6, increasing $\alpha_{\text {erc }}$ means that the average value of $F_{\text {erc }}$ becomes smaller, and the average values of $m$ and $F_{\text {comp }}$ become larger.

\section{Conclusions}

We have introduced, modeled and solved a combined vehicle routing and districting problem with stochastic customers. This problem arises in the context of courier company operations which work with a set of regular customers who are consistently serviced and also occasional customers, which must be regarded as stochastic. The problem was solved by means of a two-stage program. In the first stage, the districting decision is made. The second stage expected routing cost of each district is approximated by the Beardwood-Halton-Hammersley formula. We have developed a large neighbourhood search heuristic for the problem. Modified Solomon instances and modified Gehring and Homberger instances were used to 
assess the quality of the proposed heuristic. The computational results confirm the effectiveness of our approach.

Acknowledgments This work was partly supported by the Canadian Natural Sciences and Engineering Research Council under Grant 39682-10, by the Elite Plan Program of the Chinese National University of Defense Technology and by the Chinese National Natural Science Foundation under Grant 70971132. This support is gratefully acknowledged. Thanks are due to the Editor and to the referees for their valuable comments.

\section{References}

Applegate DL, Bixby RE, Chvátal V, Cook WJ (2006) The travelling salesman problem: a computational study. Princeton University Press

Beardwood J, Halton JH, Hammersley JM (1959) The shortest path through many points. Mathematical Proceedings of the Cambridge Philosophical Society 55(4). Cambridge University Press, 299-327

Bertsimas DJ (1992) A vehicle routing problem with stochastic demand. Oper Res 40(3):574-585

Bertsimas DJ, Jaillet P, Odoni AR (1990) A priori optimization. Oper Res 38(6):1019-1033

Blais M, Lapierre SD, Laporte G (2003) Solving a home care districting problem in an urban setting. J Oper Res Soc 54(11):1141-1147

Bozkaya B, Erkut E, Laporte G (2003) A tabu search heuristic and adaptive memory procedure for political districting. Eur J Oper Res 144(1):12-26

Carlsson JG (2011) Dividing a territory among several facilities. INFORMS J Computi. doi: 10.1287/ijoc. 1110.0479

Carlsson JG, Delage É (2011) Robust partitioning for stochastic multi-vehicle routing. Technical report of the Boeing Company.

D’Amico SJ, Wang SJ, Batta R, Rump CM (2002) A simulated annealing approach to police district design. Comput Oper Res 29(6): 667-684

Drexl A, Haase K (1999) Fast approximation methods for sales force deployment. Manag Sci 45(10): 1307-1323

Dueck G (1993) New optimization heuristics: the great deluge algorithm and the record-to-record travel. J Comput Phys 104(1): 86-92

Ferland JA, Guénette G (1990) Decision support system for the school districting problem. Oper Res 38(1):15-21

Fleischmann B, Paraschis J (1988) Solving a large scale districting problem: a case report. Comput Oper Res 15(6):521-533

Gehring H, Homberger J (1999) A parallel hybrid evolutionary metaheuristic for the vehicle routing problem with time windows. In: Miettinen K, Mäkelä M, Toivanen J (Eds.) Proceedings of EUROGEN99-short course on evolutionary algorithms in engineering and computer science, Reports of the Department of Mathematical Information Technology, Series A. Collections, No. A 2/1999. University of Jyväskylä, Finland, 57-64.

Gendreau M, Laporte G, Séguin R (1996) A tabu search heuristic for the vehicle routing problem with stochastic demands and customers. Oper Res 44(3):469-477

Goel A, Gruhn V (2008) A general vehicle routing problem. Euro J Oper Res 191(3):650-660

Groër C, Golden BL, Wasil EA (2009) The consistent vehicle routing problem. Manuf Serv Oper Manag 11(4): 630-643

Haugland D, Ho SC, Laporte G (2007) Designing delivery districts for the vehicle routing problem with stochastic demands. Euro J Oper Res 180(3): 997-1010

Hong L (2012) An improved LNS algorithm for real-time vehicle routing problem with time windows. Comput Oper Res 39(2): 151-163

Jaillet P (1988) A priori solution of a traveling salesman problem in which a random subset of the customers are visited. Oper Res 36(6): 929-936

Kalcsics J, Nickel S, Schröder M (2005) Towards a unified territorial design approach-applications, algorithms and GIS integration. TOP 13(1): 1-56

Laporte G, Louveaux FV, Mercure H (1994) A priori optimization of the probabilistic traveling salesman problem. Oper Res 42(3):543-549 
Laporte G, Louveaux FV, Van hamme L (2002) An integer L-shaped algorithm for the capacitated vehicle routing problem with stochastic demands. Oper Res 50(3):415-423

Laporte G, Musmanno R, Vocaturo F (2010) An adaptive large neighbourhood search heuristic for the capacitated arc routing problem with stochastic demands. Transp Sci 44(1):125-135

Lei H, Laporte G, Guo B (2011) The capacitated vehicle routing problem with stochastic demands and time windows. Comput Oper Res 38(12):1775-1783

Mehrotra A, Johnson EL, Nemhauser GL (1992) An optimization based heuristic for political districting. Manag Sci 44(8):1100-1114

Mendoza JE, Castanier B, Guéret C, Medaglia AL, Velasco N (2010) A memetic algorithm for the multicompartment vehicle routing problem with stochastic demands. Comput Oper Res 37(11): 1886-1898

Novaes AGN, Souza de Cursi JE, da Silva ACL, Souza JC (2009) Solving continuous location-districting problems with Voronoi diagrams. Comput Oper Res 36(1):40-59

Pisinger D, Ropke S (2007) A general heuristic for vehicle routing problems. Comput Oper Res 34(8):2403-2435

Ribeiro GM, Laporte G (2012) An adaptive large neighborhood search heuristic for the cumulative capacitated vehicle routing problem. Comput Oper Res 39(3):728-735

Ríos-Mercado RZ, Fernández E (2009) A reactive GRASP for a commercial territory design problem with multiple balancing requirements. Comput Oper Res 36(3):755- 776

Ropke S, Pisinger D (2006) An adaptive large neighborhood search heuristic for the pickup and delivery problem with time windows. Transp Sci 40(4):455-472

Shaw P (1997) A new local search algorithm providing high quality solutions to vehicle routing problems. Technical report, Department of Computer Science, University of Strathclyde, Glasgow.

Shaw P (1998) Using constraint programming and local search methods to solve vehicle routing problems. Principles and Practice of Constraint Programming-CP98, Lecture Notes in Computer Science, vol 1520. Springer, Berlin, 417-431

Skiera B, Albers S (1998) COSTA: Contribution optimizing sales territory alignment. Mark Sci 17(3):196-213

Solomon MM (1987) Algorithms for the vehicle routing and scheduling problems with time window constraints. Oper Res 35(2):254-265

Tan KC, Cheong CY, Goh CK (2007) Solving multiobjective vehicle routing problem with stochastic demand via evolutionary computation. Eur J Oper Res 177(2):813-839 